\title{
Neural Correlation Is Stimulus Modulated by Feedforward Inhibitory Circuitry
}

\author{
Jason W. Middleton, ${ }^{1,2,3}$ Cyrus Omar, ${ }^{3,4}$ Brent Doiron, ${ }^{3,5}$ and Daniel J. Simons ${ }^{1,3}$ \\ Departments of ${ }^{1}$ Neurobiology and ${ }^{2}$ Otolaryngology, University of Pittsburgh School of Medicine, Pittsburgh, Pennsylvania $15261,{ }^{3}$ Center for the Neural \\ Basis of Cognition, University of Pittsburgh and ${ }^{4}$ Carnegie Mellon University, Pittsburgh, Pennsylvania 15213, and ${ }^{5}$ Department of Mathematics, University \\ of Pittsburgh, Pittsburgh, Pennsylvania 15260
}

Correlated variability of neural spiking activity has important consequences for signal processing. How incoming sensory signals shape correlations of population responses remains unclear. Cross-correlations between spiking of different neurons may be particularly consequential in sparsely firing neural populations such as those found in layer $2 / 3$ of sensory cortex. In rat whisker barrel cortex, we found that pairs of excitatory layer $2 / 3$ neurons exhibit similarly low levels of spike count correlation during both spontaneous and sensory-evoked states. The spontaneous activity of excitatory-inhibitory neuron pairs is positively correlated, while sensory stimuli actively decorrelate joint responses. Computational modeling shows how threshold nonlinearities and local inhibition form the basis of a general decorrelating mechanism. We show that inhibitory population activity maintains low correlations in excitatory populations, especially during periods of sensory-evoked coactivation. The role of feedforward inhibition has been previously described in the context of trial-averaged phenomena. Our findings reveal a novel role for inhibition to shape correlations of neural variability and thereby prevent excessive correlations in the face of feedforward sensory-evoked activation.

\section{Introduction}

Highly variable spiking activity is a signature feature of neurons in many different areas of the brain. Variability between neurons is often correlated, as reported in visual (Kohn and Smith, 2005), auditory (Rothschild et al., 2010), and somatosensory (Greenberg et al., 2008) cortices. In many cases, excessively correlated activity is detrimental to population coding (Zohary et al., 1994; Averbeck et al., 2006; Gutnisky and Dragoi, 2008). For example, redundancy in the responses among neurons participating in a common computational task limits the degree to which variability can be reduced by population averaging. Correlated activity between neurons can be decreased through attentional modulation (Cohen and Maunsell, 2009; Mitchell et al., 2009) and active behavior (Poulet and Petersen, 2008; Gentet et al., 2010), potentially improving sensory coding. Here, we study how functional circuitry of cortical networks modulates correlations between the spiking variability of different types of neurons during spontaneous versus stimulus-evoked conditions.

In primary somatosensory (barrel) cortex, regular spike (RS) units, presumed excitatory neurons, and fast spike (FS) units, presumed inhibitory neurons, form functional networks that

Received July 7, 2011; revised Oct. 24, 2011; accepted Nov. 11, 2011.

Author contributions: J.W.M., B.D., and D.J.S. designed research; J.W.M., C.O., and B.D. performed research; J.W.M. analyzed data; J.W.M., B.D., and D.J.S. wrote the paper.

This work is supported by NSF Grant DMS-081714 (B.D.) and NIH Grant NS-19950 (D.J.S). J.W.M. was supported by a Canadian Institutes of Health Research postdoctoral fellowship, and C.O. is supported by a Department of Energy Computational Science Graduate Fellowship.

We thank T. Tzounopoulos and L. Maler for careful reading of this manuscript.

Correspondence should be addressed to Dr. Jason W. Middleton, Department of Neurobiology, University of Pittsburgh School of Medicine, 200 Lothrop Street, BST E1407, Pittsburgh, PA 15261. E-mail: jmiddlet@pitt.edu.

DOI:10.1523/JNEUROSCI.3474-11.2012

Copyright $\odot 2012$ the authors $\quad 0270-6474 / 12 / 320506-13 \$ 15.00 / 0$ process whisker stimulus information (Bruno and Simons, 2002; Swadlow, 2003). Local cortical interconnections are dense (Yoshimura and Callaway, 2005; Oswald et al., 2009), suggesting that, on average, individual pairs of cortical neurons in these functional networks share a significant portion of their synaptic inputs. To reveal how functional coupling modulates network responses, we recorded from pairs of neurons (RS-RS, FS-RS, and FS-FS) in layer 2/3 (L2/3) somatosensory cortex; these neurons fire sparsely (O'Connor et al., 2010) and receive the bulk of their inputs from other cortical neurons. To study the variability of their joint activity, we compared spike count correlations between pairs of neurons during both spontaneous and whisker stimulus-evoked states. Theoretical work predicts that the increase in firing rates associated with a whisker deflection causes an increase in coincident firing as well as an increase in the correlation coefficient of spiking activity (de la Rocha et al., 2007). Contrary to this prediction, we find that the correlation coefficient between the spike counts from RS and FS neurons decreases when stimulus-driven joint firing activity increases.

Our single-unit data show that the variability of FS and RS population spontaneous activity is weakly correlated by a background synaptic field. We use this finding to develop a computational model of interacting inhibitory and excitatory populations whose variable activity is strongly dependent on common fluctuating inputs. Our firing rate model reproduces key experimental findings only if population activation functions are nonlinear and only when the excitatory population receives feedforward inhibition. Together, our results suggest that feedforward inhibition helps maintain low levels of correlated variability of spiking activity despite elevated, evoked firing due to feedforward sensory-driven excitation. Thus, feedforward inhibition, in addi- 
tion to maintaining temporal precision (Pinto et al., 1996; Higley and Contreras, 2006) and increasing the dynamic range of sensory information coding (Pouille et al., 2009), also maintains low levels of correlation leading to improved population coding.

\section{Materials and Methods}

Animal preparation. Data were obtained from 13 Sprague Dawley adult female rats. Surgical procedures and maintenance of rats during recording sessions, approved by the University of Pittsburgh Institutional Animal Care and Use Committee, are similar to those previously described (Bruno and Simons, 2002). Briefly, rats were anesthetized with isoflurane, and a tracheal tube was inserted to maintain a clear air passage. Small-diameter Silastic tubing was inserted into the external jugular vein for drug delivery, and a small Teflon catheter was inserted into the right femoral artery for monitoring blood pressure. The skull was exposed, and small stainless-steel screws were inserted into the bone over the contralateral occipital and frontal lobes for electrocorticogram (ECoG) monitoring; an additional screw was inserted into the bone over the ipsilateral frontal lobe to serve as a reference for cortical microelectrode recordings. The skull was thinned by careful drilling, and a small area of bone $\left(<0.5 \times 0.5 \mathrm{~mm}^{2}\right)$ overlying the right barrel cortex was removed. Saline was periodically applied to an acrylic dam constructed around the craniotomies.

Isoflurane was discontinued during the recording session and the rat was maintained in a lightly narcotized state using fentanyl (Baxter Healthcare; $\left.10 \mu \mathrm{g} \cdot \mathrm{kg}^{-1} \cdot \mathrm{h}^{-1}\right)$. Spontaneous and whisker-evoked firings are comparable with those observed in awake, undrugged animals (Simons et al., 1992). The rat was immobilized with pancuronium bromide (SICOR Pharmaceuticals; $1.6 \mathrm{mg} \cdot \mathrm{kg}^{-1} \cdot \mathrm{h}^{-1}$ ) to prevent spontaneous whisker movements that could otherwise interfere with the use of our whisker stimulators (below). Body temperature was maintained at $37^{\circ} \mathrm{C}$ using a servo-controlled heating blanket (Harvard Apparatus). Blood pressure, heart rate, tracheal airway pressure, and ECoG were monitored throughout the recording session with a personal computer using custom-written software. If any of these indicators could not be maintained within normal physiological ranges, the experiment was terminated.

Upon termination of an experiment, the rat was deeply anesthetized with sodium pentobarbital $(100 \mathrm{mg} / \mathrm{kg}$, i.v. $)$ and transcardially perfused (2\% paraformaldehyde and $1.5 \%$ glutaraldehyde in $0.1 \mathrm{~m}$ phosphate buffer) for cytochrome oxidase (CO) histochemistry. The cortex was cut tangentially (60 $\mu \mathrm{m}$ sections), and sections were reacted for CO (Land and Simons, 1985) and were counterstained with thionine. Using microdrive readings, signs of tissue disruption, and electrolytic lesions made during the experiment, recording sites were localized with respect to individual underlying barrels. In cases in which electrode tracks could not be discerned with certainty, interlesion distance in underlying sections and distance between placements of electrodes on the pia were used to estimate interelectrode distance.

Whisker stimulation. Whiskers were deflected using a custom-built piezoelectric stimulator (Simons, 1983) attached $10 \mathrm{~mm}$ from the base of the whisker. Stimulus waveforms, stored on disk, were output at $10 \mathrm{kHz}$ via an eight-channel, fast digital-to-analog converter. Whiskers were randomly deflected $1 \mathrm{~mm}$ in one of eight directions $\left(0,45,90^{\circ}\right.$, etc.) using a "ramp-and-hold" stimulus. The ramp phase of the deflection was $\sim 8 \mathrm{~ms}$ long, with a mean velocity of $125 \mathrm{~mm} / \mathrm{s}$. The whisker deflection was maintained for $200 \mathrm{~ms}$, and the whisker was then returned to its resting or neutral position with the same speed as the initial deflection. Each randomized block of eight stimuli were repeated 40 times for a total of 320 whisker deflections. Individual stimuli were presented one per $\sim 4 \mathrm{~s}$.

Electrophysiology. Simultaneous recordings were obtained using a multichannel Eckhorn matrix (MM-5; Thomas Recording). Platinum/ iridium in quartz fibers ( $60 \mu \mathrm{m}$ diameter) were pulled and ground to $2-5$ $\mu \mathrm{m}$ tip diameters, having impedances of 1-6 M $\Omega$. Microelectrodes were advanced one-by-one into L2/3. The principal whisker (PW) of a cortical neuron was defined as the whisker whose deflection evokes the largest spike response, relative to other whiskers. Two or three electrodes were used in each recording session. These were placed $\sim 100-200 \mu \mathrm{m}$ apart centered over the underlying layer 4 barrel as determined beforehand using micromapping procedures. The vast majority of recorded pairs had the same PW; data from a small number of pairs in which the PWs were different were not included in the analyses.

Microelectrode signals were bandpass filtered $(300 \mathrm{~Hz}$ to $10 \mathrm{kHz})$ and passed to a personal computer where spike waveforms were detected in real time using custom programmed acquisition software (LabVIEW; National Instruments) and then stored for further analysis. In some experiments, local field potentials (LFPs) were recorded on one of the electrodes in place of single-unit activity. LFP signals were bandpass filtered $(0.1 \mathrm{~Hz}$ to $9 \mathrm{kHz})$ and sampled at $32 \mathrm{kHz}$. Signals were later downsampled to $1 \mathrm{kHz}$ for further analysis.

The state of arousal of the rats was assessed by on-line monitoring of LFP recordings and the ECoG. When the LFP and ECoG were dominated by slow, large-amplitude events, indicating that the animal was in a sleeplike state, data were not collected. These states sometimes occurred toward the end of long recording sessions ( $>6 \mathrm{~h}$ ). In all cases, if a state of light sedation could not be maintained for extended periods of time with the prescribed dosage of fentanyl $\left(10 \mu \mathrm{g} \cdot \mathrm{kg}^{-1} \cdot \mathrm{h}^{-1}\right)$, the experiment was terminated.

Unit sampling. Spike waveforms were analyzed with cluster analysis using custom programmed software in LabVIEW. FS and RS unit spike waveforms are typically distinct, the former being longer in duration (see Fig. 1A). After sorting, mean spike waveforms were calculated and the duration of early and late components of the waveforms were measured. A two-dimensional scatterplot of these two components reveals two clusters (Bruno and Simons, 2002), and cell type identity was assigned based on this criteria. Individual, well isolated units were recorded on different electrodes of the multielectrode array. In no case was more than one unit taken from a single microelectrode.

The data set consists of 48 FS-RS pairs and 31 RS-RS pairs studied during spontaneous and stimulus-evoked conditions. A smaller sample of 9 FS-FS unit paired recordings was also obtained. For a separate set of 11 FS-RS pairs, the units were recorded simultaneously during spontaneous firing conditions with concurrent LFPs. Within the stimulated data set (see Figs. 1-4), 5 FS units are common to two different RS units, 3 RS units are common to two different FS units, and 15 RS units are common to two different RS units. This gives 49 unique FS units and 71 unique RS units. Adding the 11 unique FS-RS pairs recorded for spontaneous activity (see Fig. 5) gives a complete data set that is comprised of 60 unique FS units and 82 unique RS units.

Data analysis. The raw, or trial-matched, joint poststimulus histogram (jPSTH) is defined as follows:

$$
\operatorname{jPSTH}\left(t_{i}, t_{j}\right)=\sum_{k} \delta_{1}^{k}\left(t_{i}\right) \delta_{2}^{k}\left(t_{j}\right),
$$

where $\delta_{1,2}^{k}\left(t_{i}\right)$ is the $k$ th trial of the spike train of neuron 1 or 2 . To quantify the frequency of coincidences above chance level, we subtract a trial-shuffled version of the jPSTH from the trial-matched version, giving the shuffle-corrected jPSTH.

Correlation coefficients were calculated in a standard fashion using Pearson's correlation coefficient as follows:

$$
\rho_{X Y}=\operatorname{cov}(X, Y) / \sigma_{X} \sigma_{Y},
$$

where $X$ and $Y$ are the signals of interest and $\sigma$ denotes their SDs. Correlation coefficients were calculated using 320 trials of paired responses to whisker deflection. Correlation coefficients were first calculated using the 40 trials for a specific deflection angle, and then these values were averaged over all eight angles $\left(0,45, \ldots, 315^{\circ}\right)$.

Recordings of spontaneous spiking activity (13 min average duration) were obtained from 11 FS-RS-pairs while simultaneously recording an LFP from a nearby electrode $(\sim 100-200 \mu \mathrm{m})$. Spike train signals for the pairs, $X_{\mathrm{FS}}$ and $X_{\mathrm{RS}}$, were created from recorded spike times. The crosscorrelation function was calculated directly as follows: $C_{\mathrm{FS}-\mathrm{RS}}(\tau)=$ $\left\langle X_{\mathrm{FS}}(t) \cdot X_{\mathrm{RS}}(t+\tau)\right\rangle$ for each pair, and then averaged over 11 pairs. This direct estimate was compared with an indirect estimate of the crosscorrelation using independent correlations of the LFP with each spike train. Spike-triggered average (STA) LFPs were obtained for each unit of 
the pair as follows: $\operatorname{STA}_{\alpha}(t)=\Sigma_{i} \operatorname{LFP}\left(t-t_{j}^{\alpha}\right)$, where the subscript $\alpha=\mathrm{FS}$, RS denotes either the fast-spike or regular-spike population, respectively, and $t_{j}^{\alpha}$ are the spike times. Assuming that the correlation between FS and RS units is driven by a common synaptic input, of which the LFP is reflective, the cross-correlation function can be rewritten as follows:

$$
\begin{aligned}
C_{\mathrm{FS}-\mathrm{RS}}(\tau) & \cong\left\langle N_{\mathrm{FS}}\right\rangle \\
\cdot & \left\langle N_{\mathrm{RS}}\right\rangle F^{-1}\left[\frac{\mathrm{STA}_{\mathrm{FS}}(f)^{\star} \tilde{\mathrm{STA}}_{\mathrm{RS}}(f)}{\tilde{\sigma}^{2}(f)}\right],
\end{aligned}
$$

where ST̃A denotes the Fourier transform of the spike-triggered average, $\tilde{\sigma}^{2}$ is the power of the LFP, and $F^{-1}$ is the inverse Fourier transform operator. $N_{\mathrm{FS}}$ and $N_{\mathrm{RS}}$ are the number of FS and $\mathrm{RS}$ spikes recorded in a trial. The asterisk $\left(^{*}\right)$ indicates a complex conjugate operation. This "partial spectra" method was previously used to predict cross-correlations in both basal ganglia and cortex (Goldberg et al., 2004).

The signal-to-noise ratio quantifies the ratio of signal strength, $R$, to the SD of single-unit trial-to-trial fluctuations, $d$. For a population of size $N$ with mean pairwise correlation, $\rho$, the SNR is given by the following (Zohary et al., 1994):

$$
\mathrm{SNR}=\frac{N \cdot R}{\sqrt{N d^{2}+N(N-1) \rho d^{2}}} .
$$

A
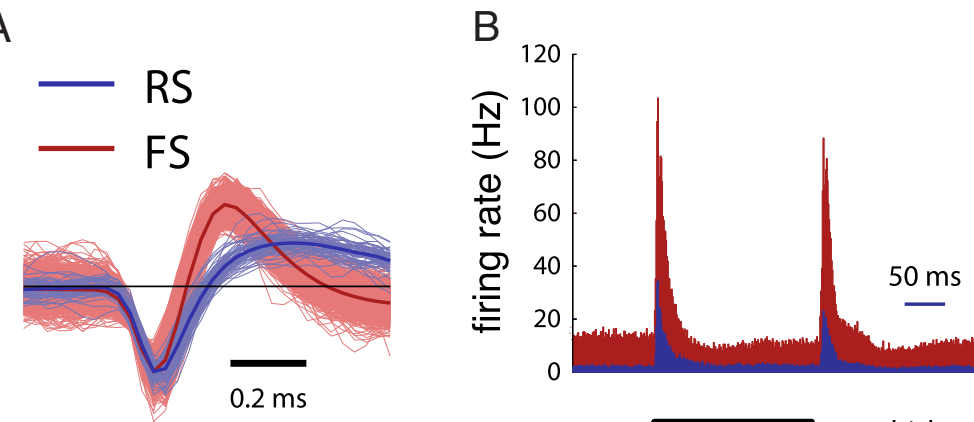

C
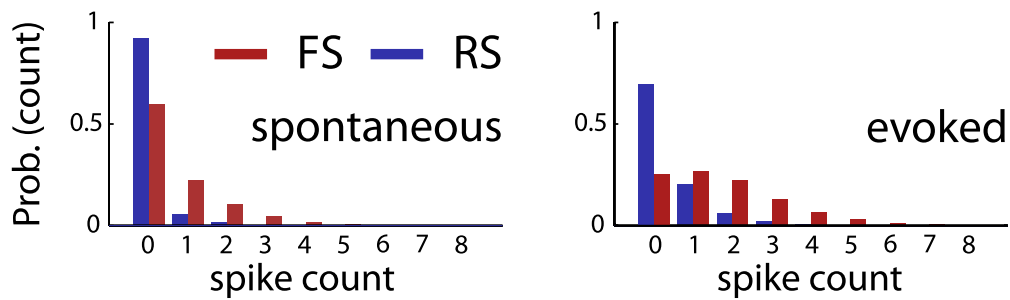

Figure 1. Variability of whisker-driven activity of neurons in L2/3. $A$, FS and RS neurons were classified according to the time course of their extracellular spike waveforms, shown here for an example pair of neurons (see Materials and Methods). $\boldsymbol{B}$, FS and RS PSTHs displayed a rapid increase and slower relaxation of the average firing rate in response to whisker deflection onset; responses to stimulus offset have a similar time course but were smaller in magnitude $(n=48)$. C, The probability densities of spontaneous spike counts ( $50 \mathrm{~ms}$ time window) revealed temporally sparse activity, particularly for RS units (left). Whisker stimulus resulted in larger and more variable spike counts (right).
All statistical analyses were performed using custom programs in the MATLAB (MathWorks) environment. One-way ANOVAs, followed by pairwise $t$ tests, were used to test significance.

Modeling. The model is based on one previously used to describe trialaveraged (deterministic) dynamics of the thalamocortical feedforward circuit of the barrel cortex (Pinto et al., 1996). Population firing rates of excitatory and inhibitory neural populations are governed by the following equations:

$$
\begin{aligned}
& r_{\mathrm{FS}}(t)=f_{\mathrm{FS}}\left[\mu_{\mathrm{FS}}+W_{\mathrm{FS}}(t)+\eta_{\mathrm{FS}}(t)\right] \\
& r_{\mathrm{RS}}(t)=f_{\mathrm{RS}}\left[\mu_{\mathrm{RS}}+W_{\mathrm{RS}}(t)-g I(t)+\eta_{\mathrm{RS}}(t)\right],
\end{aligned}
$$

where $r_{\mathrm{FS}}(t)$ and $r_{\mathrm{RS}}(t)$ are time-dependent firing rates of FS and RS populations, respectively, and $g$ is inhibition strength. $\mu_{\mathrm{FS}, \mathrm{RS}}$ are the input biases to each model; these parameters set the average depolarization relative to the threshold nonlinearity. The whisker-evoked synaptic input, $W_{\alpha}(t)$, is given by the following:

$$
W_{\alpha}(t)=\left[\begin{array}{cc}
0 & , \quad t<t_{0} \\
A_{\alpha}\left(\frac{t-t_{0}}{3}\right)^{-3 / 2} e^{-1.65 /\left(t-t_{0}\right)} & , \quad t \geq t_{0}
\end{array},\right.
$$

where $t_{0}$ is the time of the whisker deflection and $A_{\alpha}$ is the amplitude of the excitatory synaptic drive, the subscript $\alpha=$ FS, RS indicating cell type. Synaptic drive represents the population-averaged excitatory drive from layer 4 (L4) cells on to L2/3 cells. The form of Equation 6 qualitatively reproduces the temporal evolution of the whisker-evoked response from L4 (Pinto et al., 1996).

The fluctuating inputs, $\eta_{\alpha}$, were low-pass-filtered Gaussian white-noise processes. The frequency cutoff of the filter was chosen to be $12 \mathrm{~Hz}$, which qualitatively captures the slow timescales seen in LFP-STAs (see Fig. 5B). These dynamic processes have zero mean and an autocorrelation given by the following: $\left\langle\eta_{\alpha}(t) \cdot \eta_{\alpha}(t \quad+\quad \tau)\right\rangle=$ $\sigma_{\alpha} e^{-|t| \tau_{\alpha}}$. The correlation coefficient between $\eta_{\mathrm{FS}}$ and $\eta_{\mathrm{RS}}$ was set to $c$. For simplicity, the population model is unidirectionally coupled, as illustrated in Figure $6 A$, with the RS population receiving FS inhibition (Eq. 5). The out- put firing rate of the inhibitory population is filtered by the following synaptic dynamics:

$$
\tau_{I} \frac{d I}{d t}=-I(t)+r_{\mathrm{FS}}(t)
$$

resulting in an inhibitory synaptic input, $I$, to the RS-population of strength, $g$. The synaptic inputs to each population are transformed into output firing rate, $f_{\alpha}$, by a threshold-linear firing rate transfer function as follows:

$$
f_{\alpha}(x)=\max \left\{0, K_{\alpha} x\right\} .
$$

The parameters used for the model results, unless otherwise indicated, are as follows: $g=0.0076, c=0.714, \mu_{\mathrm{FS}, \mathrm{RS}}=0.37,0.12, A_{\mathrm{FS}, \mathrm{RS}}=3.05$, $1.45, \tau_{\mathrm{FS}, \mathrm{RS}}=83 \mathrm{~ms}, \sigma_{\mathrm{FS}, \mathrm{RS}}=0.566,0.172, \tau_{I}=3 \mathrm{~ms}$, and $K_{\mathrm{FS}, \mathrm{RS}}=30$. Simulations of Equations 5-8 were performed with a stochastic Euler integration scheme $(\Delta t=0.05 \mathrm{~ms})$.

The timescale of the background fluctuations, $\tau_{\mathrm{FS}, \mathrm{RS}}$, is much longer than the timescale of the whisker input and cortical response. This permits the approximation $\eta_{\alpha}$ by a random variable whose equilibrium density is as follows: $p\left(\eta_{\mathrm{FS}}, \eta_{\mathrm{RS}}\right)=N\left(0, \sigma_{\mathrm{FS}}^{2}, \sigma_{\mathrm{RS}}^{2}, C\right)$ (i.e., zero mean, normally distributed random variables with variances $r_{\mathrm{FS}}=f_{\mathrm{fS}}\left(\mu_{\mathrm{FS}}+\right.$ $\left.W_{\mathrm{FS}}+\eta_{\mathrm{FS}}\right)$ and correlation coefficient $c$ ). To further simplify calculations, we set the inhibitory timescale, $\tau$, to zero. In total, we have a mapping of the stationary random variables $r_{\mathrm{FS}}=f_{\mathrm{FS}}\left(\mu_{\mathrm{FS}}+W_{\mathrm{FS}}+\eta_{\mathrm{FS}}\right)$ and $r_{\mathrm{RS}}=f_{\mathrm{RS}}\left(\mu_{\mathrm{RS}}+W_{\mathrm{RS}}+\eta_{\mathrm{RS}}-g r_{\mathrm{FS}}\right)$. Standard techniques for mapping random variables (Papoulis and Pillai, 2002) were used to obtain analytic expressions for the joint density $P\left(r_{\mathrm{FS}}, r_{\mathrm{RS}}\right)$. Numerical quadrature formulas were used to solve for the first and second moments of $P\left(r_{\mathrm{FS}}, r_{\mathrm{RS}}\right)$. These techniques were used for the data in Figures 8 and 9.

\section{Results}

\section{Layer 2/3 spiking activity and spontaneous correlations}

Extracellular spike recordings from multiple single units in L2/3 somatosensory cortex were recorded on a multielectrode array (one unit per electrode) (see Materials and Methods). Cluster analysis on the temporal features of spike waveforms (Fig. 1A) 

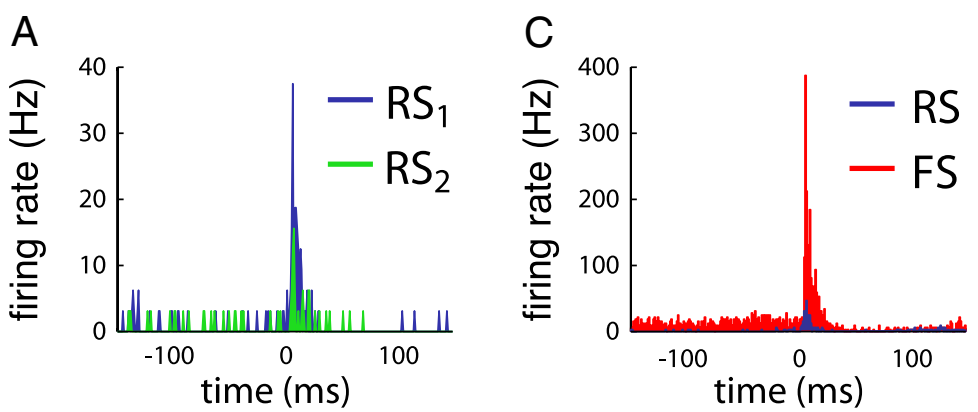

B

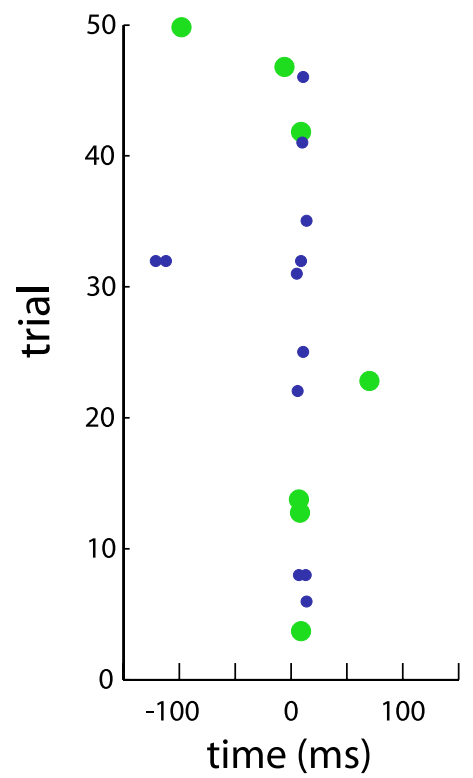

D

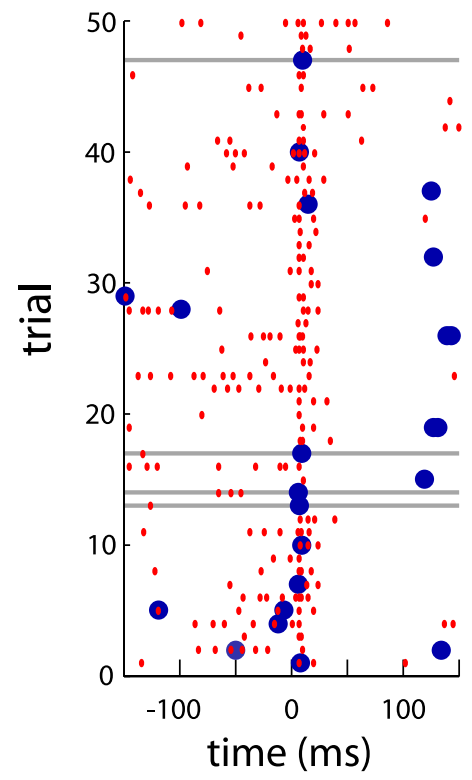

Figure 2. Variability of joint responses in L2/3. A, Average PSTHs for a pair of simultaneously recorded RS units in response to a whisker deflection. Whisker deflection onset is at $0 \mathrm{~ms}$. $\boldsymbol{B}$, Raster plots of the spike times for the pair of RS units shown in $\boldsymbol{A}$. There were no trials in which the RS units fire coincident spikes in during either spontaneous activity or during whisker deflections. $\boldsymbol{C}$, Average PSTHs for a pair of simultaneously recorded FS and RS units in response to whisker deflection. $D$, In the 150 ms preceding whisker deflection onset, every RS unit spike occurred at nearly the same time as an FS spike. However, in response to whisker deflections, there were some trials in which the RS unit fired and the FS did not ( 4 of 9 trials; indicated by gray bars).

permitted cell type classification. RS unit waveforms had longer positive and negative phases compared with those from FS units (Fig. 1A). Whisker deflections caused rapid and transient increases in both FS and RS unit average firing rates (Fig. $1 B$ ). Similar, yet smaller, transients were evoked in response to deflection offsets (Fig. 1B). FS and RS cells fired spontaneously at $13.6 \pm 1.3$ and $2.1 \pm 0.6 \mathrm{~Hz}$, and had peak stimulus-evoked firing rates of $103 \pm$ 12 and $35 \pm 5 \mathrm{~Hz}$, respectively. Average responses faithfully encoded changes in the whisker deflection angle. Spike count histograms revealed that firing on individual trials was sparse (Fig. 1C). Although the probability of spiking remained below 50\% per deflection, the probability of firing one or more spikes did increase significantly above spontaneous values immediately after whisker deflection (Fig. 1C). A qualitatively similar effect was seen for FS units; however, in more than one-half of trials FS units fired one or more spikes after whisker deflection.

Given the sparse nature of neural firing in L2/3, it is important to know the extent to which paired activity is coordinated on a trial-to-trial basis. Figure $2 \mathrm{~A}$ shows the trial-averaged PSTHs for two simultaneously recorded RS units centered on the time of a whisker deflection onset. A raster plot of the spike activity on individual trials reveals that these two units did not fire together either before the stimulus or during the stimulus (Fig. 2B). This suggests that these two RS units were firing independently. Figure $2 C$ shows the trial-averaged PSTHs for simultaneously recorded FS and RS units. On individual trials, whenever the RS unit fired in the prestimulus period, the FS unit also fired at nearly the same time (Fig. 2D). However, during the stimulus response, the FS unit was silent in nearly one-half of the trials that the RS unit spiked (four of nine; Fig. 2D). These findings suggest that FS and RS units fire in a relatively coordinated fashion during spontaneous activity and their firing becomes more independent with whisker stimulation.

To quantify these observations, we calculated the shuffle-corrected jPSTH of simultaneously recorded neurons (see Materials and Methods). The shufflecorrected jPSTH gives the number of coincident spikes above or below chance levels. The average FS-RS jPSTH is positive along the diagonal, revealing that FS-RS activity was positively correlated (Fig. 3A; $n=48$ ). Consistent with the example in Figure $2 D$, the population FS-RS jPSTH dips to negative values during the whisker deflection onset (Fig. $3 B$ ). The RS-RS jPSTH is near zero (Fig. $3 C$; $n=31$ ) and changes relatively little during whisker stimulation (Fig. 3D), consistent with our observation that RS units fired relatively independently of each other (Fig. 2B). We also recorded from a smaller number of FS-FS pairs $(n=9)$ and found that they too fired relatively independently of each other (Fig. $3 E$ ), with a brief period of increased joint activity during the stimulus (Fig. $3 F$ ). Thus, cells of similar type fire relatively independently during both spontaneous and stimulus-evoked conditions, whereas FS-RS populations become decorrelated upon whisker deflection. For the remainder of this manuscript, we will focus both on RS-RS correlations because they are the primary output of L2/3, and on FS-RS correlations for their role in overall firing decorrelation.

To account for possible changes in spiking variability that may occur during stimulus-evoked periods, we used Pearson's correlation coefficient to examine spike counts (see Materials and Methods). This measure has the dual advantage of being normalized by the spike count variance and of allowing us to use a counting window large enough to compensate for low firing rates. The spike count correlation is commonly used to study the correlation structure of spiking activity in sensory systems (de la Rocha et al., 2007; Smith and Kohn, 2008; Ecker et al., 2010; Renart et al., 2010). The temporal sequence of FS-RS correlation coefficient values (using a sliding $50 \mathrm{~ms}$ time window) revealed a close relationship between the onset of elevated firing and the decorrelation of trial-to-trial variability (Fig. $4 A$, middle). In contrast, RS-RS correlation did not depend on the time course of whisker-evoked activation (Fig. $4 A$, top). The average correlation coefficient of RS-RS pair spontaneous activity was $0.04 \pm 0.03$, and the average correlation of joint FS-RS spontaneous activity 
A

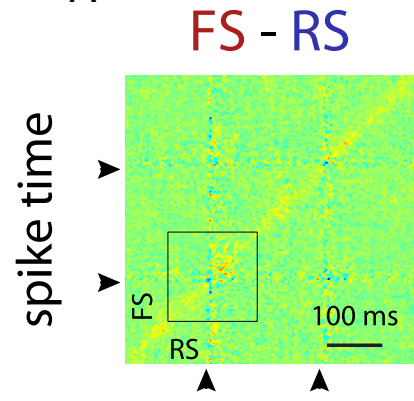

B

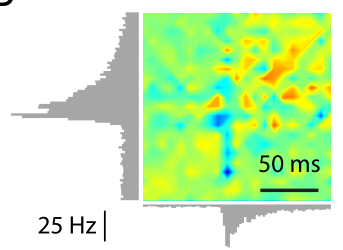

C

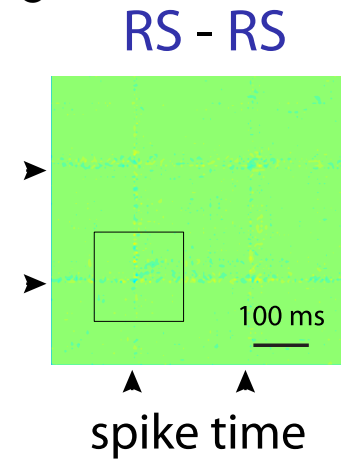

D

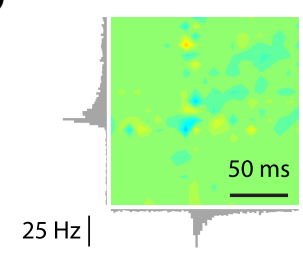

E

FS - FS

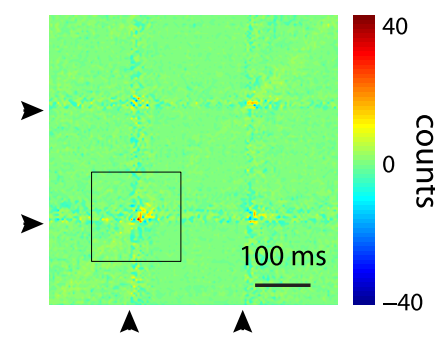

$\mathrm{F}$

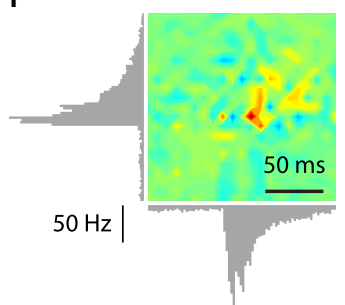

Figure 3. Correlation structure of neurons in L2/3. A, The shuffle-corrected jPSTH revealed a nonzero band along the positive diagonal, indicating that spontaneous FS and RS activity was correlated above chance levels. Whisker deflection onset and offset times are indicated by arrowheads. $\boldsymbol{B}$, An expanded view of the jPSTH around deflection onset (indicated by square in $\boldsymbol{A}$ ) revealed a brief desynchronization of FS and RS activity. C, D, The shuffle-corrected RS-RS jPSTH was uniformly low and did not change much during whisker stimulation. $E$, $\boldsymbol{F}$, Similarly, FS-FS pairs exhibited near zero correlation except for a short time after whisker deflection onset.

was $0.11 \pm 0.02$ (Fig. $4 B$ ). When whiskers were deflected the correlation coefficient was reduced for FS-RS pairs $\left(\rho_{\mathrm{FS}-\mathrm{RS}}=\right.$ $0.01 \pm 0.01 \mathrm{SEM}, n=48, t_{(47)}=5.69, p<$ 0.001 , paired $t$ test) but not for RS-RS pairs (Fig. $4 B$; $\rho_{\mathrm{RS}-\mathrm{RS}}=0.02 \pm 0.01 \mathrm{SEM}$, $n=31, t_{(30)}=0.6150, p=0.54$, paired $t$ test). In total, whisker deflections decorrelated FS-RS activity, whereas RS-RS correlations were uniformly low throughout both spontaneous and stimulus-evoked periods.

PSTHs of RS activity conditioned on the level of FS spontaneous activity immediately preceding a stimulus illustrate dependence between FS and RS variability. The trials with the highest spontaneous FS activity (measured in the $50 \mathrm{~ms}$ preceding stimulus onset) corresponded to the lowest RS stimulus-evoked responses (Fig. $4 C$, dark blue). This trend reversed with the lowest FS spontaneous firing rates being followed by high RS responses (Fig. 4C, light blue). This conditional dependence suggests an active decorrelation mechanism that is dependent on the ongoing, spontaneous L2/3 population activity.

\section{Correlated population fluctuations} during spontaneous activity

To understand network level mechanisms underlying decorrelation of FS-RS activity, we examined their correlations with field recordings in L2/3. Over large spatial scales, synaptic fluctuations

A

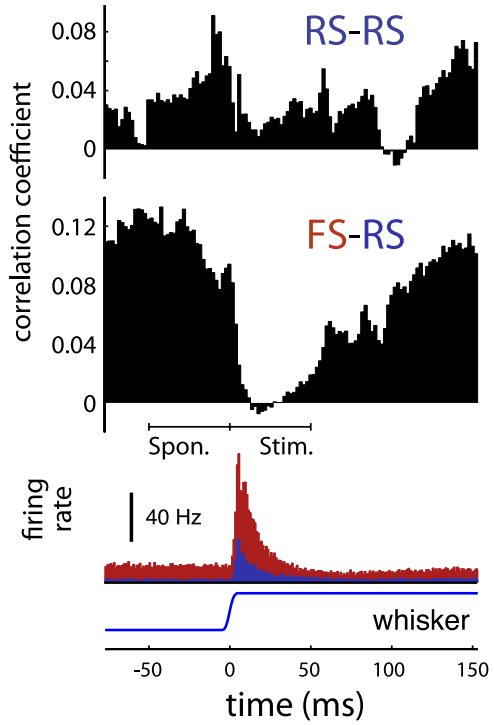

B

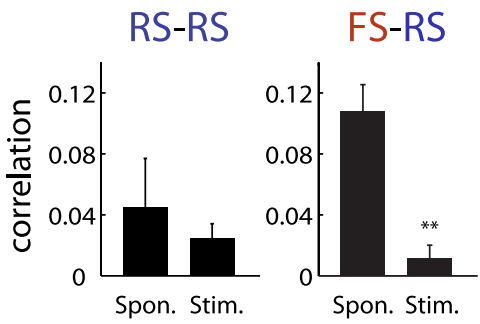

C

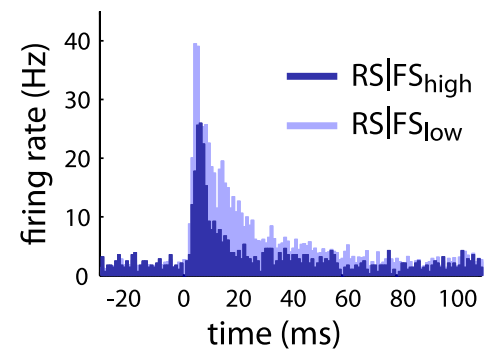

Figure 4. FS and RS spiking is decorrelated during whisker deflection. $A$, The time course for RS-RS correlation coefficient (top; $n=31$ pairs), calculated using $50 \mathrm{~ms}$ sliding time windows in $2 \mathrm{~ms}$ increments. There was no apparent dependence of the time course of RS-RS correlation on whisker evoked population activity (bottom). A clear decrease in FS-RS correlation (middle; $n=48$ pairs) was observed when their joint stimulus-evoked activity increases (bottom). The correlation then returns toward prestimulus values. $B, \mathrm{RS}-\mathrm{RS}$ correlation is similarly small during spontaneous and stimulus-evoked periods (left). FS-RS correlation was significantly reduced upon whisker deflection $\left({ }^{* *} p<0.001\right)$. There are no significant differences in the mean RS-RS correlation coefficients (top) when comparing any to two periods $(p=0.5375)$. The period over which correlations are averaged to obtain reported values are indicated by the horizontal bars below the middle panel in $A$. Error bars indicate SEM. C, When the RS PSTH was conditioned on the preceding spontaneous FS activity, an inversely proportional relationship was observed. High spontaneous FS activity leads to low RS responses (dark blue), while low spontaneous FS activity leads to higher RS responses (light blue).

are represented in the variation of LFPs (Kreiman et al., 2006). Common synaptic inputs can act to correlate spike activities of two neurons (de la Rocha et al., 2007) and thus may give rise to the observed correlation of FS and RS spontaneous activity. LFPs 


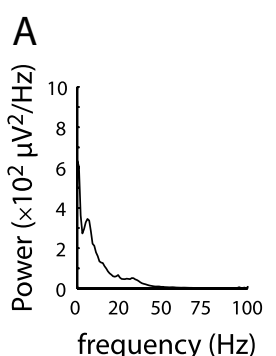

B STA

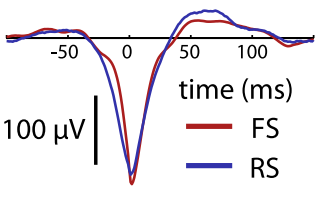

C

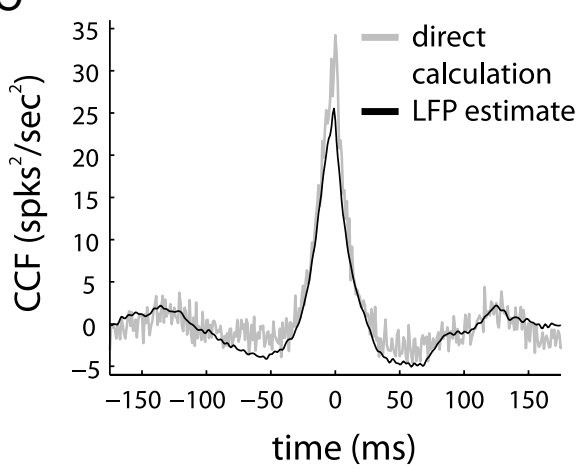

D

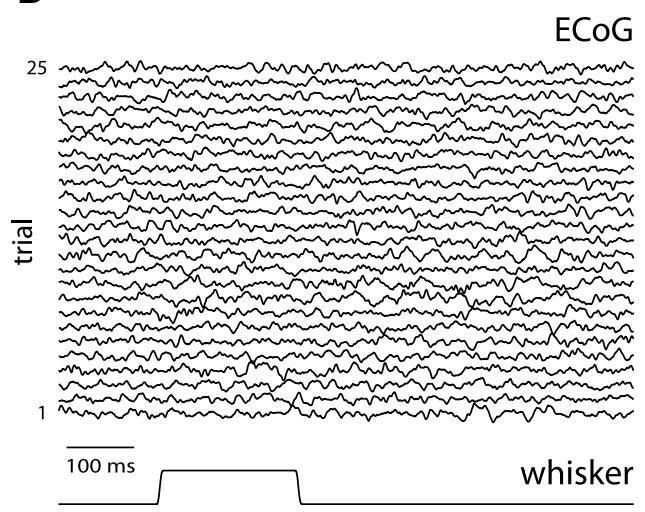

Figure 5. Correlated synaptic fields account for spike correlations in the spontaneous state. $A$, The average power spectrum of spontaneous (no stimulation) local field potential activity ( $n=11) . \boldsymbol{B}$, FS and RS unit STAs with respect to the ongoing LFP. C, The CCF of FS-RS spontaneous activity estimated indirectly via their independent correlation with the LFP (black line) agreed well with the direct calculation of the CCF (gray line). $D$, An example of EC $0 G$ recordings during multiple trials of stimulus presentation. Whisker stimulus deflection onset and offset (bottom) do not induce a change in the state of the ECOG.

may predict correlations between neurons as they represent common inputs. We therefore recorded spontaneous activity from 11 FS-RS pairs along with the LFP from a separate nearby electrode $(\sim 100-200 \mu \mathrm{m})$. The LFP power spectrum reveals that low frequencies contribute to a large portion of LFP fluctuations (Fig. $5 A$ ), consistent with LFP recordings in primary visual cortex (Berens et al., 2008), as well as spectral analysis of membrane voltage fluctuations in the barrel cortex of rats maintained under conditions nearly identical with those used here (Constantinople and Bruno, 2011). To examine how ongoing spiking activity of L2/3 neurons correlated with the LFP, we computed STAs of the LFP for FS and RS units (Fig. 5B). An estimate of the cross-correlation function (CCF) between FS and RS units was obtained indirectly by using LFP-STAs (Goldberg et al., 2004) (see Materials and Methods). The estimate agreed well with the true CCF, computed directly from FS-RS spiking activity (Fig. 5C). Furthermore, the components of the LFP that correlated neural activity were predominately slow, indicated by the large temporal width of the predicted CCF. Thus, the slow components of the LFP represent, to a large degree, a common synaptic field that correlates neural populations under spontaneous conditions, in agreement with studies in visual cortex (Kelly et al., 2010) and basal ganglia (Goldberg et al., 2004). It should be noted that this analysis was performed on recordings of spontaneous activity. While a direct comparison of the CCF in Figure $5 C$ to the correlation analyses of whisker-evoked activity in Figures 3 and 4 is difficult, it can be seen that the structure of the CCF is qualitatively similar to the profile of the FS-RS jPSTH along the antidiagonal (Fig. $2 \mathrm{~A}$ ).

A change in behavioral state, accompanied by a decrease in low-frequency power of the background component of the LFP, could cause a decorrelation in the FS-RS spiking activity. Whisker deflections were presented at intervals of $\sim 4 \mathrm{~s}$, and it is unlikely that the state of cortical network changed systematically during interstimulus periods. Nevertheless, to ensure that results were not confounded by behavioral state changes, we monitored ECoG signals throughout the experiments. No changes were observed in the ECoG after whisker stimulation (Fig. 5D). Any potential sensory-evoked changes in arousal would arise through indirect pathways (Constantinople and Bruno, 2011) and likely be delayed much longer than the stimulus response duration.

\section{Population models}

The experimental results motivate a computational model of FS and RS populations driven by slow common fluctuations. We adapted a population model of feedforward inhibitory circuitry, used previously to reproduce trial-averaged firing rates of FS and RS unit activity (Pinto et al., 1996), to model the trial-to-trial variability of L2/3 FS and RS populations (see Materials and Methods). Specifically, we modeled FS and RS activity with population average firing rates. The RS population received monosynaptic inhibition, of strength $g$, from the FS population (Fig. $6 A)$. Both populations received excitation from feedforward, trial-locked simulated whisker-related inputs from L4 (Fig. 6A, labeled $W$ ). Finally, because sensory cortical neurons exhibited sharp input-output transfer threshold nonlinearities (Carandini, 2004), we incorporated this feature into the model populations. Each model population received its own fluctuating input current (Fig. $6 \mathrm{~B}$, left). The transfer functions are linear rectifiers with an output threshold relative to the mean input current (Fig. $6 B$, middle). The portions of the input below threshold are not transferred to the output so that output firing rates arise from the net sum of currents above threshold (Fig. $6 \mathrm{~B}$, right). Thus constructed, the model accurately described the instantaneous firing rates of the two populations, during both spontaneous and evoked activity (Fig. 7, red and blue traces).

To quantify the transfer of correlations in this model system, two values are calculated: the input correlation, $\rho_{\text {in }}$, and the output correlation, $\rho_{\text {out }}$. The input correlation, $\rho_{\text {in }}$, is the correlation coefficient of the net sum of all the inputs to the population rate transfer functions (Fig. $6 \mathrm{~B}$, left). The output correlation, $\rho_{\text {out }}$, is the correlation coefficient of the population firing rates, $r_{\mathrm{FS}}(t)$ and $r_{\mathrm{RS}}(t)$ (Fig. 6B, right). These correlations are calculated on a point-by-point basis and thus measure trial-by-trial variability as a function of time. Note that $\rho_{\text {in }}$ is not, by definition, the same as $c$. Whereas $c$ is a model parameter representing correlation of the fluctuating background inputs, $\rho_{\text {in }}$ is a measured value that quantifies correlations arising from all inputs. In our full model, the inhibition from the FS to the RS population is also a source of 
variability in the input currents, and hence $\rho_{\text {in }}$ will depend on both $c$ and $g$ (see Materials and Methods).

We next examined how key model features (i.e., local inhibition, threshold nonlinearities, and correlated inputs) determined the correlation of trial-totrial variability. We analyzed how the correlation of input currents, $\rho_{\text {in }}$, was transformed into the correlation of output rates, $\rho_{\text {out }}$. As noted above, feedforward inhibition results in differences in the common field correlation $c$ and the total RS-RS $\rho_{\text {in }}$. We are interested in how this difference, combined with the thresholding of RS population activity, shapes the RS-RS output correlation $\rho_{\text {out }}$.

In the absence of both feedforward inhibition and threshold nonlinearity (i.e., an uncoupled, linear model), $\rho_{\text {in }}$ was equal to $c$ during spontaneous conditions because there was no coupling. Furthermore, the linear transfer resulted in $\rho_{\text {out }}=$ $\rho_{\text {in }}=c$ (de la Rocha et al., 2007), even while the firing rates of the model populations were elevated by whisker deflection (Fig. 7A, top). When a threshold was added to the neural transfer, the spontaneous output correlation was lower $\left(\rho_{\text {out }}\right.$ $<\rho_{\text {in }}$ ), and stimulus-induced activation increased $\rho_{\text {out }}$ to match that of the linear model (Fig. 7A, bottom).

When feedforward inhibition, of strength $g$, was included (i.e., the FS population inhibits the RS population), $\rho_{\text {out }}$ decreased significantly for both linear and nonlinear models, compared with their uncoupled counterparts (Fig. $7 B$, thick and dashed lines). However, the nonlinear model captured both the spontaneous and stimulus-evoked decorrelation of FS-RS activity (Fig. 7B, thick line) observed in our experiments (Fig. 4), while the linear model did not (Fig. $7 B$, dashed line). To understand how recurrent excitation acts to shape correlations in this model, we added an excitatory connection, of strength $g_{e}=$ 0.0076, from the RS to the FS population. With this additional feature, the baseline spontaneous correlation is higher, yet relative stimulus-induced decorrelation is still observed as in the inhibition-only model (Fig. $7 B$, thin line). Together, these findings suggest that a combination of threshold nonlinearity and feedforward inhibitory circuitry is necessary and sufficient to account for the stimulus-evoked decorrelation of FS-RS activity.

Note that the nonlinear model produces a sharp increase in correlation that immediately transitions into the longer period of decorrelation. This effect is due to the dynamics of the FS-to-RS synapse (Eq. 7), which introduces a very short lag before the inhibition can act to decorrelate FS-RS responses. This transient increase is not observed in our data. We believe that we would observe this transient effect if correlations for spike counts with a $1 \mathrm{~ms}$ precision could be confidently estimated from our current data set. Currently, we use a $50 \mathrm{~ms}$ window to generate spike counts, which gives us on average $\sim 530$ FS unit spikes and $\sim 150$ RS unit spikes with which to estimate the correlation coefficient for each pair. If a $1 \mathrm{~ms}$ counting window is used, on average, $\sim 3$ RS unit spikes and $\sim 10$ FS spikes are available to estimate the correlation coefficient between one FS-RS pair.
To further understand the influence of inhibition and threshold nonlinearities on $\rho_{\text {in }}$ and $\rho_{\text {out }}$, we examined the quasi-static approximation of our model. This effectively treats FS and RS activities as static random variables, as opposed to stochastic processes (see Materials and Methods), ignoring temporal nonstationarities of FS and RS activity arising from either the time course of the whisker deflection or synaptic integration. As a validation of this approach, we first observed that with weak inhibition, FS-RS correlation increased with greater whiskerrelated feedforward input, $W$ (Fig. $8 A$ ). This agreed with the uncoupled dynamic model results (Fig. 7A). Furthermore, with strong inhibition, larger inputs decorrelated FS-RS variability, also matching our dynamic model (Fig. 7B).

The quasi-static approximation permitted the derivation of stationary probability densities of joint FS-RS inputs and joint FS-RS output activity (see Materials and Methods). The relationship between $\rho_{\text {in }}$ and $\rho_{\text {out }}$ can be visualized by plotting the twodimensional probability density of inputs to the FS and RS units with respect to the output thresholds (Fig. $8 \mathrm{~B}$ ). In this representation, the portion of the input distribution in the gray region is subthreshold and does not contribute to the response distributions. Rather, only the portion in the first quadrant is in the superthreshold, which corresponds to the output joint distribution (Fig. $8 B$, right). When inputs were positively correlated (as evidenced by the eccentricity along the positive diagonal; left), output thresholds that rectify inputs resulted in more weakly correlated output variables (i.e., $\rho_{\text {out }}<\rho_{\text {in }}$; Fig. $8 B$, right).

We next used the input and output joint densities to examine how the correlation mapping from $\rho_{\text {in }}$ to $\rho_{\text {out }}$ of the FS-RS model depended on $c$ and $g$ for levels of excitation corresponding to 

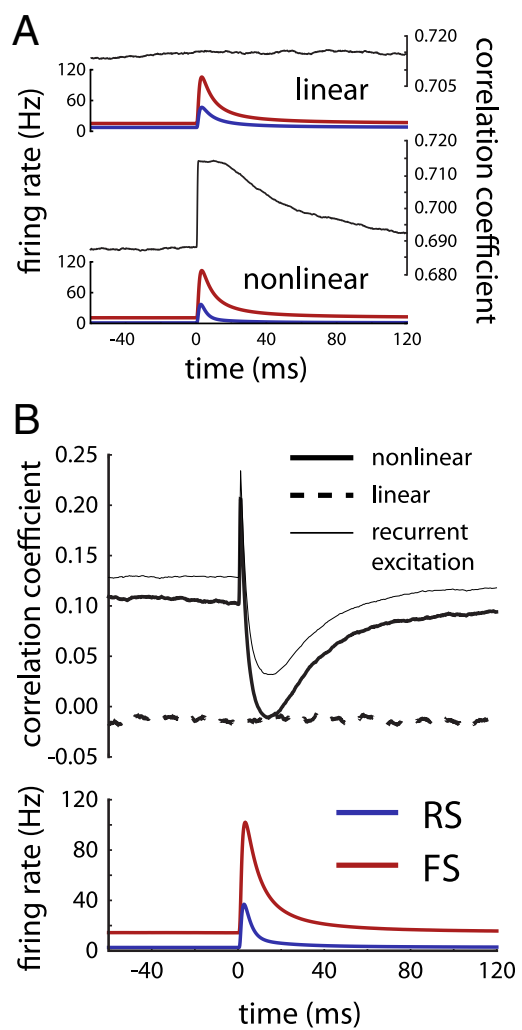

Figure 7. A simple feedforward inhibitory model describes stimulus-induced decorrelation. $A$, The effect of stimulus on output correlation, in the uncoupled model, depended on the output threshold nonlinearity. In a linear system, with no output threshold, stimulus-evoked activation of population firing rates did not induce a change in correlation (top). When the populations have a nonlinear output rate transfer, there was a stimulus-evoked increase in correlation concurrent with increases in population firing rates (bottom). $\boldsymbol{B}$. When the nonlinear model included inhibitory coupling, excitatory feedforward stimulus-evoked input still increased the population firing rates (bottom) but now decreased the correlation of the variability of these rates (thick line; top). When the transfer nonlinearity was removed, the correlation of FS and RS activity was stimulus independent and uniformly low (dashed line; top). Recurrent excitation from the RS to the FS population results in an upward shift of the correlation coefficient; however, the stimulus-induced decorrelation remains (thin line; top).

spontaneous and evoked states. Some input into the uncoupled model with correlated inputs $(c>0 ; g=0)$ was subthreshold, resulting in a lower output versus input correlation (Fig. $8 C$, top left, black region). In the evoked state, the input density was elevated above threshold (Fig. $8 C$, bottom left), so that $\rho_{\text {out }}=\rho_{\text {in }}$. In contrast, a model with uncorrelated inputs and inhibitory coupling $(c=0 ; g>0)$ exposed the anticorrelating effects of inhibition so that $\rho_{\text {in }}<0$, apparent from the negatively sloped eccentricity of the input distribution (Fig. $8 C$, top middle). In the spontaneous state, the thresholds attenuated the transfer of this negative correlation to the output in a similar fashion to the uncoupled model. In the evoked state, the inhibition had a larger decorrelating effect (smaller $\rho_{\text {in }}$; Fig. $8 C$, bottom middle). The entire input density was above threshold, yielding $\rho_{\text {out }}=\rho_{\text {in }}$. Finally, in a model with both correlated background inputs $(c>$ $0)$ and inhibitory coupling $(g>0)$, the opposing influence of $c$ and $g$ sets $\rho_{\text {in }}$ to an intermediate, positive value in the spontaneous state (Fig. $8 C$, top right). FS units were more linear and their firing was more variable in the evoked state, thereby enhancing the decorrelating effects of inhibitory fluctuations. This permitted a more even balance between the positively correlating effects of the common inputs and the anticorrelating effects of feedfor- ward inhibition, resulting in an overall decorrelated evoked state (Fig. $8 C$, bottom right; -0.052 vs 0.071 ).

To explore the balance between background correlations and local circuit-mediated anticorrelations, we jointly varied $g$ and $c$, obtaining a spontaneous state FS-RS correlation that matches experimental values over a broad range of $(g, c)$ pairings (Fig. 9, dashed line). A similarly broad range of $(g, c)$ parameter pairs yielded FS-RS correlation that matched experimentally observed stimulus-evoked values (Fig. 9, solid line). However, the lines intersect only at one point; this corresponds to the $(g, c)$ pair that reproduced the qualitative differences in both spontaneous and stimulus-evoked output correlations (Fig. $8 \mathrm{C}$, right). We note that the intersection point gave a $(g, c)$ pair that implied both a strong background correlation and strong inhibitory coupling. This predicts that, whereas weakly correlated background fluctuations and weak feedforward inhibition can reproduce either the spontaneous or evoked states, independently, only strong correlated fluctuations and strong inhibition can simultaneously reproduce the output correlation in both states.

Reported levels of FS-to-RS connection probability (Yoshimura and Callaway, 2005; Oswald et al., 2009) make it likely that many of our recorded pairs are not directly synaptically coupled. However, the success of the population model in reproducing pairwise stimulus-induced decorrelation does not require each FS-RS pair to have a direct disynaptic inhibitory connection. The slow decay of noise correlations over space (Kerr et al., 2007; Rothschild et al., 2010) is consistent with the assumption of our model that, in addition to direct coupling, common global inputs also dictate to pairwise correlations. As FS units within the population covary, to some degree, they will all act on RS units in a similar fashion. Thus, an uncoupled FS-RS pair becomes decorrelated through the action of another, unobserved FS unit that is coupled to the RS cell.

In summary, feedforward disynaptic inhibition and input correlation have opposing influences on the output correlation of FS and RS neural populations. The balance of these two forces is controlled by the level of the excitation of the cells relative to their transfer function threshold nonlinearities. Hence, the degree of cancellation of anticorrelated inputs is not ongoing but is dependent on the level of network activation.

\section{Globally correlated inhibition decorrelates RS-RS responses}

While whisker deflections decorrelated trial-to-trial variability of FS-RS activity, RS-RS correlation was uniformly low for both spontaneous and whisker-evoked periods (Fig. 4A). To explain how the mechanism underlying inhibitory-mediated FS-RS decorrelation determines the level of RS-RS correlation, we expanded our model to include a separate, second pair of FS-RS populations (Fig. 10A). Here, each FS-RS population pair represents a separate functional unit. The populations (FS and RS) within a functional unit retain their original degree of fluctuating background correlation $\left(c_{\text {intra }}=0.714\right)$. Populations across functional units share fluctuations with weaker correlations $\left(c_{\text {inter }}=\right.$ 0.146), reflecting a more global, less strongly coupled network (Fig. $10 \mathrm{~A}$, bottom). $c_{\text {inter }}$ was set to reproduce experimentally observed RS-RS spontaneous correlation.

Upon whisker deflection, RS-RS firing becomes transiently more correlated followed by a longer-lasting, shallow decrease in correlation, reaching a low of $\rho=0.028$ (Fig. $10 B$, cyan). We compared this condition to a model in which the correlation of background inputs between the two FS populations is removed (i.e., $c_{\text {inter; }}^{\mathrm{FS}}$ Fig. $10 \mathrm{~A}$, top), resulting in independently fluctuating inhibitory currents into the RS populations. In this case, the cor- 

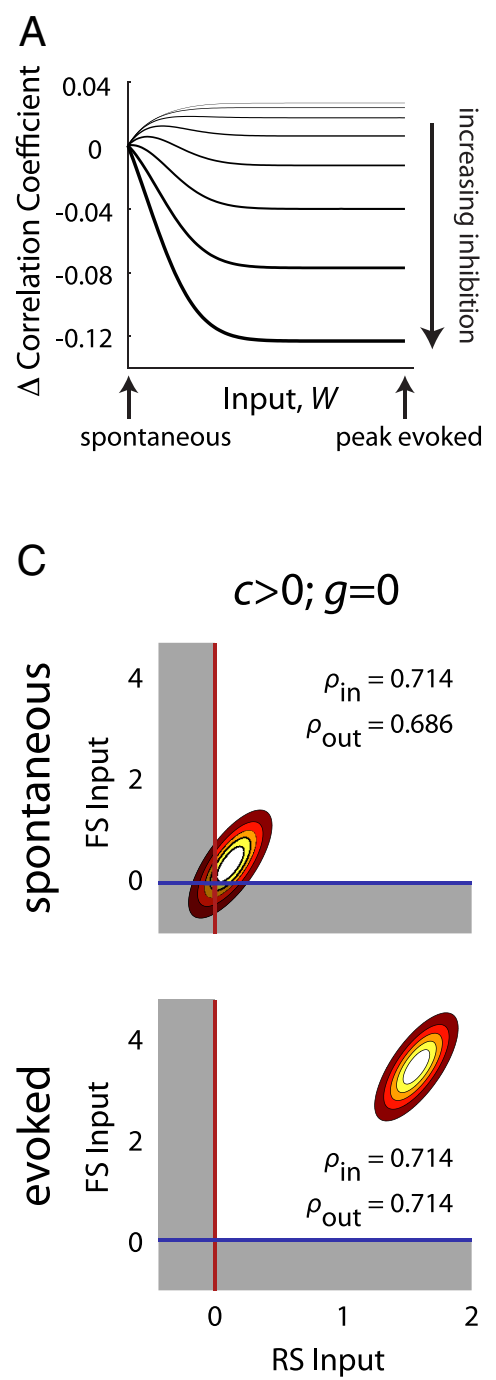

B

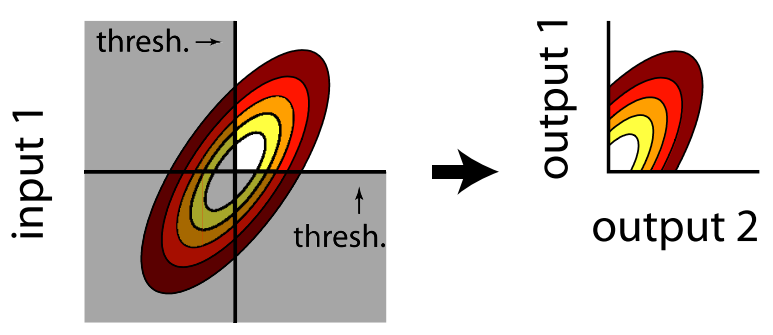

input 2 $c>0 ; g>0$
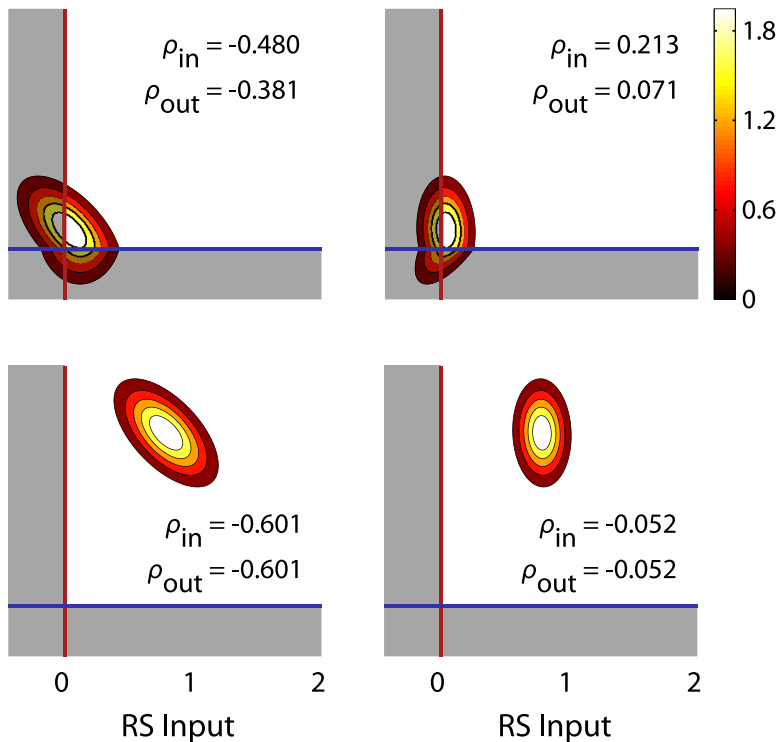

Figure 8. A threshold-dependent balance of inhibitory coupling and background input correlation was required for stimulus-evoked output decorrelation. $\boldsymbol{A}$, The change in output correlation, relative to spontaneous values as whisker input, $\boldsymbol{W}$, was increased, showed that larger inhibitory coupling strength (thin to thick lines) enhanced the anticorrelating effect of average input drive. $\boldsymbol{B}$, The effect of input- output thresholds on correlation is illustrated by the example of a model with positively correlated inputs. A large eccentricity of the input distribution along the positive diagonal indicates positive correlation (left). Thresholds bisect the inputs, resulting in an output distribution with a smaller eccentricity (right), and thus smaller output correlation coefficient. $\boldsymbol{C}$, In a model system with common inputs and no coupling, the spontaneous output correlation, $\rho_{\text {out }}$, was smaller than the input correlation, $\rho_{\text {in }}$ (left top). In the stimulus-evoked regime, the input and output correlations were the same (left bottom). $\rho_{\text {out }}$ was less negative than $\rho_{\text {in }}$ for the uncoupled model with inhibition, in the spontaneous state (middle top). In the evoked state, the output correlation was equal to the large negative input correlation (middle bottom). A model with both coupling and inhibition (right column) transitioned from a weakly correlated spontaneous state to an uncorrelated state evoked state. The input axes for all panels incorporate all inputs including background fluctuations, feedforward whisker inputs, and feedforward inhibition.

relation of spontaneous RS-RS activity was higher, and whisker deflection resulted in a long-lasting increase in correlation (Fig. $10 \mathrm{~B}$, yellow), peaking at $\rho=0.288$. These values contrast with our experimental RS-RS correlations (Fig. 4A). As an additional comparison, we reduced the input correlation between RS populations to $c_{\text {inter }}^{\mathrm{RS}}=0.029$ (keeping $c_{\text {inter }}^{\mathrm{FS}}=0$ ) to match the experimental spontaneous RS-RS correlations $\left(\rho_{\text {spont }}=0.04\right)$. Still, a small stimulus-driven increase in output correlation remained, peaking at $\rho=0.054$ (Fig. $10 \mathrm{~B}$, green). In total, these findings show that in the same way that inhibition, driven by globally coherent synaptic fluctuations, decorrelates FS-RS population activity, it also acts to prevent an increase in correlation of RS-RS population activity associated with stimulus presentation.

The functional impact of different values of correlation on information processing can be quantified in a straightforward manner by calculating the signal-to-noise ratio (SNR) of an RS population response to a graded stimulus (see Materials and
Methods). The ratio $\mathrm{SNR}_{N} / \mathrm{SNR}_{1}$ gives the relative SNR improvement for a population of size $N$ relative to a single neuron. Higher SNR values for larger populations are achieved through a reduction of noise fluctuations through population averaging. The SNR ratios are shown for the peak stimulus-driven model correlations (Fig. 10C). For large populations, the model with globally coherent inhibition performs 3.2 times better than the model with independently fluctuating inhibition and 1.4 times better than the model with uncorrelated inhibition and lowered background input correlations to the RS populations. As shown previously, small differences in correlation of neural activity can have disproportionately large effects on the ability of a population to represent neural information due to their inverse scaling relationship (Zohary et al., 1994). Therefore, coherent feedforward inhibitory fluctuations, through their decorrelating effect on RS-RS variability, lead to improved population rate-based representation of signals. Recent advances in optogenetic engi- 


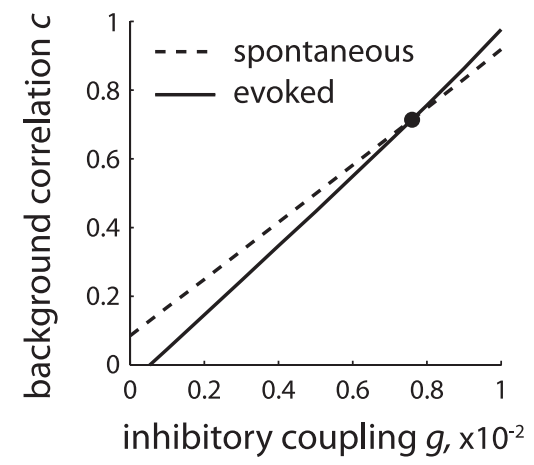

Figure 9. Unique model parameter pairs lead to spontaneous and evoked correlations consistent with experimental recordings. A continuum of inhibition, $g$, and background input correlation, $c$, values resulted in an invariant correlation coefficient for output model variability (dashed line). A different continuum of parameter pair values results in a fixed evoked correlation coefficient (solid line). The intersection of the two parameter sets (black dot) specified a balanced pair of inhibition, $g$, and background correlation, $c$, values that satisfied observed output correlations in both spontaneous and evoked states.

neering have introduced the stable step function opsin (SSFO) (Yizhar et al., 2011). This form of channelrhodopsin can introduce long-lasting tonic depolarizing currents into select excitatory or inhibitory neural populations and shift the excitatory/ inhibitory balance of local network activity. Using this novel stimulating technique to tonically bias the activity of inhibitory neurons to higher values would likely result in a lower trial-totrial spike count correlation in pairs of excitatory neurons in sensory cortices. We predict that biasing the activity level of FS units in somatosensory or visual cortex during a sensory stimulus discrimination task, using SSFOs, would impair an animal's performance.

Our modeling efforts relied on neural populations receiving correlated background synaptic inputs. We based this model feature on our observations of spike coherence with a slowly fluctuating LFP in a lightly anesthetized preparation. Slow oscillatory network dynamics occur in whisker barrel cortex of behaving animals. The coherence between spiking activity and the LFP depends on behavioral motivation while the amplitude of the LFP remains constant (Ganguly and Kleinfeld, 2004). During rewarded whisking conditions, spike-LFP coherence was larger than during unrewarded conditions. We expect that under conditions of large spike-LFP coherence, whisking-related network oscillations would contribute to population activity decorrelation in the same way the global background fluctuations we measure do.

\section{Discussion}

The role of the variability of neural activity in neural computation is a topic receiving increasing focus. Variability is not simply detrimental "noise" that interferes with cognition and sensory processing, but instead is intimately connected with behavioral output (M. M. Churchland et al., 2006; A. K. Churchland et al., 2011). It is important to understand how neural circuitry, previously considered to dictate trial-averaged phenomena, contributes to the properties of variable population activity. Furthermore, it is important to understand how the same circuitry affects correlations of the variability of neural activity.

The disynaptic feedforward inhibitory circuit, a canonical cortical circuit (Douglas et al., 2004), consists of direct excitation and disynaptic inhibition, both driven by the same afferent input. Disynaptic inhibition plays a critical role in maintaining tempo- rally precise responses to excitation (Berman and Maler, 1998; Pouille and Scanziani, 2001; Pinto et al., 2003; Wehr and Zador, 2003; Higley and Contreras, 2006; Heiss et al., 2008), modulating the gain of sensory information (Lewis and Maler, 2002; Kanichay and Silver, 2008), promoting neural synchrony and oscillations (Galán et al., 2006; Bartos et al., 2007), and shaping receptive field properties (Ferster and Miller, 2000; Miller et al., 2001). Despite our broad knowledge about the influence of this canonical circuitry on trial-averaged temporal responses, relatively little is known about its influence on trial-to-trial variability and correlation of spiking activity.

We examined how disynaptic feedforward inhibitory coupling shapes correlated variability between the spiking activity of excitatory and inhibitory neurons. We found that paired spike recordings from inhibitory and excitatory neurons in $\mathrm{L} 2 / 3$ of the whisker-related primary somatosensory cortex exhibited levels of correlation that differed during spontaneous and stimulusevoked states. The firing of FS units and RS units were positively correlated under spontaneous conditions. Both FS and RS spiking activities covaried with slow fluctuating components of LFPs indicating that correlated spontaneous FS-RS firing exists on long timescales and is due to common synaptic drive. When whiskers were deflected, evoked FS and RS units were jointly activated, but the trial-to-trial variability of this response was uncorrelated despite the fact that both FS and RS cells were presumably driven by the same afferents. Evidence suggests that balanced recurrent excitation and inhibition acts to reduce correlations (Renart et al., 2010). We extend these findings by showing that feedforward inhibition plays an important role in decorrelating population activity during sensory stimulation.

Numerical simulations and calculations of equilibrium states in a minimal feedforward FS-RS model showed that strong correlating background activity, feedforward inhibitory architecture, and intrinsic spike threshold nonlinearities were sufficient to account for both the spontaneous and evoked data. Our results suggest that local circuit neural firing is weakly correlated, reflecting opposing forces of strong LFP-induced synchronization and of strong FS-mediated anticorrelating effects. Such anticorrelating effects of inhibition also ensure that excitatory (pyramidal) cells fire independently, enhancing population coding of sensory stimuli.

In this study, we compared the correlation of spontaneous activity to the correlation of responses to whisker deflections of only one velocity. We showed that feedforward excitation from whisker deflections increased both the correlating effects of the background synaptic field and the anticorrelating effects of the feedforward inhibition. We predict that these two opposing influences will be balanced over a wide range of whisker deflection velocities so that RS-RS correlations will be maintained at low levels in a stimulus-independent manner. While the results of our uncoupled model (Fig. 6A) are consistent with a previous theoretical framework establishing how correlations are transferred through uncoupled networks (de la Rocha et al., 2007), our study builds on that understanding by showing how inhibitory coupling further modulates the transfer of correlations in a feedforward network architecture.

We incorporated the effects of a background field simply by adding subthreshold fluctuating synaptic inputs into both populations. The origin of this global field, however, remains unclear. Slow spontaneous fluctuations could arise from feedforward effects of synaptic coupling (Doiron et al., 2006) or may arise from direct thalamocortical projection to L2/3 (Petreanu et al., 2009). Slow spontaneous waves of activity (Ferezou et al., 2007) have 

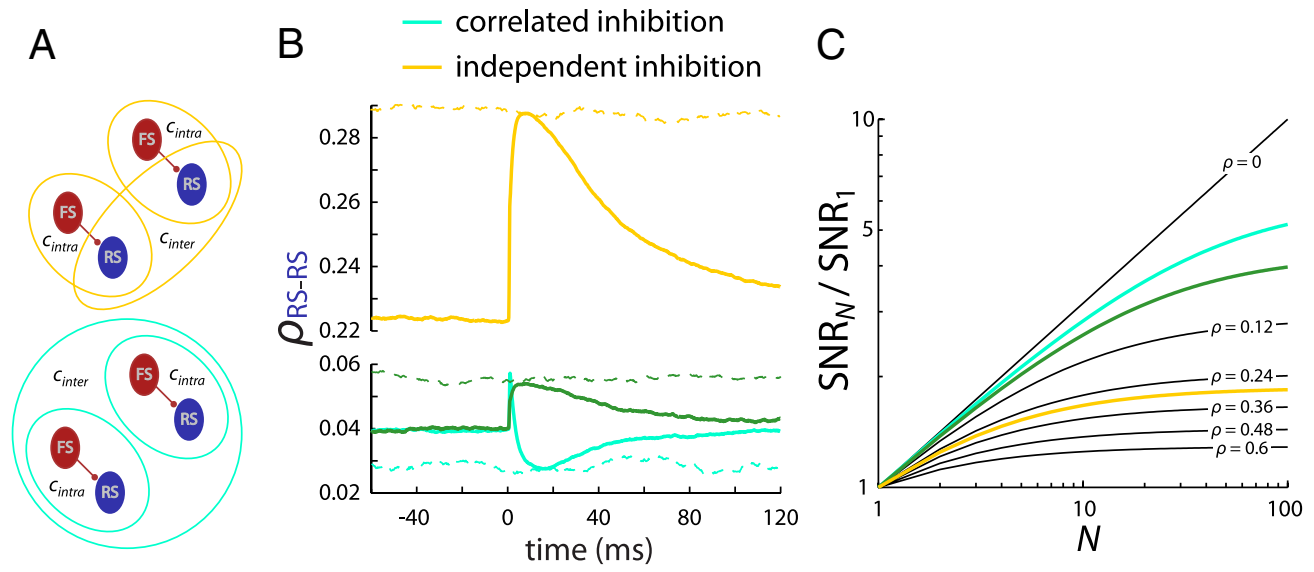

Figure 10. Low stimulus-evoked RS-RS correlation was consistent with a cancellation of input correlations by correlated inhibition. $A$, An expansion of our model included a second functional unit consisting of an FS-RS population pair. The FS-RS input correlation for each population was the same as before, $c_{\text {intra }}=0.714$, but in addition, both pairs receive more global, yet weaker, correlated inputs $\left(c_{\text {inter }}=0.146\right)$. Two variants of this model were ones in which the FS populations receive common inputs $\left(c_{\text {inter }}^{\mathrm{FS}}=0.146\right.$; cyan $)$ or alternatively received inputs that were uncorrelated from one another $\left(c_{\text {inter }}^{\mathrm{FS}}=0\right.$; yellow), resulting in independently fluctuating inhibition. $\boldsymbol{B}$, When the FS populations received globally correlated inputs $\left(c_{\text {inter }}^{\mathrm{FS}}=0.146\right.$; cyan), there was a low spontaneous RS-RS correlation and a small stimulus-evoked decrease in $\rho_{\mathrm{RS}-\mathrm{RS}}$. When $c_{\text {inter }}^{\mathrm{FS}}=0$ (yellow), RS-RS spontaneous correlation was higher and there was a stimulus-evoked increase in correlation. A further decrease of the background input correlation between RS populations ( $\left.c_{\text {inter }}^{\mathrm{RS}}=0.029\right)$ yielded a spontaneous correlation that was similar to the case in which inhibition was globally coherent, but a stimulus-evoked increase in RS-RS output correlation (green) was still observed. Linear versions of the models with and without coherent inhibition (dashed cyan and yellow curves, respectively) result in constant, stimulus-independent values of correlation. Also, the linear version of the model without coherent inhibition and reduced $c$ is stimulus independent (dashed green). These values correspond to the peak stimulus-induced correlation of their respective nonlinear versions. $C$, The population signal-to-noise ratio (relative to a single neuron) was enhanced as the population size, $N$, increases. In the limit of large $N$, the system with globally coherent inhibition performs 3.2 and 1.4 times better than the system with independent inhibition and independent inhibition with lowered RS input correlation, respectively. SNR curves for six other evenly spaced correlation coefficients (black) are shown for reference.

been shown to modulate stimulus-evoked responses in sensory cortices (Arieli et al., 1996; Petersen et al., 2003). Trial-to-trial sensory responses in the visual system are positively correlated with spontaneous population activity (Arieli et al., 1996), while in the somatosensory system RS unit responses were found to be inversely proportional to instantaneous voltage-sensitive dye recordings (Petersen et al., 2003). We extend these findings by showing that the spontaneous network state differentially modulates responses of FS and RS populations and that this interdependent response is a result of feedforward inhibitory circuitry.

Pairwise correlations arising from a spatially broad synaptic field permitted the description of population activity using a simple population firing rate model (Ermentrout, 1998). Firing rate models, of either single-unit or population activity, have been used to describe a number of trial-averaged effects [e.g., contrast invariance (Anderson et al., 2000), establishing integration windows (Pinto et al., 1996), and the generation of waves of cortical activity (Huang et al., 2004)]. Furthermore, it has been shown that firing rate threshold nonlinearities can qualitatively reproduce trial-to-trial variability of single-unit responses (Carandini, 2004). Here, we show that a simple rate model also successfully captures the correlation structure of population activity in a feedforward, disynaptic inhibitory circuit.

Many studies have developed an understanding of the role of inhibition in neural synchronization (Börgers and Kopell, 2003; Tiesinga et al., 2004; Brunel and Hansel, 2006; Cardin et al., 2009). The mechanism of synchronization involves direct reciprocal connections between excitatory and inhibitory neurons, which can drive gamma rhythms that are observable in the LFP (Mazzoni et al., 2008). In contrast, our study is consistent with the recent proposal that asynchronous cortical states (Ecker et al., 2010) are maintained, in part, by inhibition (Renart et al., 2010). These two putative roles of inhibition seem to be in direct opposition to one another. However, a resolution to this seeming incongruity is possible by considering the state of the network that measured neurons are embedded in. In our study and that of
Renart et al. (2010), both inhibitory and excitatory populations of neurons receive external commons sources of fluctuations. These common fluctuations engage both excitatory and inhibitory populations in such a way as to cancel overall input correlations. However, this mechanism requires a balance between excitation and inhibition. When the excitatory-inhibitory balance is shifted, high-frequency rhythmic network synchronization is induced (Brunel, 2000; Börgers and Kopell, 2003; Yizhar et al., 2011). Changes in behavioral state may engage or disengage the functional architecture in such a way as to upregulate or downregulate excitatory-inhibitory balance. While we do not observe strong LFP power at gamma frequencies (Fig. $5 \mathrm{~A}$ ), it is possible that gamma rhythms would emerge with more complex ongoing whisker stimulation. With the low firing rates we observe, it is possible that gamma networks can be established at the level of population activity (Brunel, 2000). However, large-scale recording techniques would be needed to confirm this. Increased subject attention has been shown to reduce low-frequency spike count correlations (Cohen and Maunsell, 2009; Mitchell et al., 2009) in the same cortical areas that show increased highfrequency gamma oscillations (Fries et al., 2001; Chalk et al., 2010). Thus, it is still likely that slow modulations of the firing rates of FS units would still be able to decorrelate spike counts of RS units on long timescales in the presence of faster gamma oscillations.

We believe that inhibition-mediated decorrelation of neural activity is possible in less sparsely firing systems. Low correlations have been observed in visual (Ecker et al., 2010), auditory, and somatosensory (Renart et al., 2010) neural population responses. A similar decorrelating role for inhibition was proposed to explain the low values of correlation in these data sets (Renart et al., 2010). Correlations of spike responses in V4 are lower when their receptive field is attended to (Cohen and Maunsell, 2009; Mitchell et al., 2009). Attention also increases firing rates of both RS and FS units in the same area (Mitchell et al., 2007). Taking these two results together, we predict that simultaneously recorded FS and 
RS units would exhibit lower correlation in a state of attention, thus strengthening our proposal for a state-dependent decorrelating role of inhibition.

While afferent firing synchrony is essential for propagation of sensory signals along successive stages in an afferent pathway (Bruno and Sakmann, 2006), excessive correlation can be detrimental to population coding (Averbeck et al., 2006). Purely excitatory feedforward circuits amplify synchronous spike discharge (Reyes, 2003). Our findings thus suggest an additional function of feedforward inhibitory circuits, namely, the suppression of excessive correlation of spiking within functionally related groups of neurons. Furthermore, we found that stimulus-evoked decorrelation depends on the level of afferent drive. This shows that the control of correlated activity is a dynamic process that enables cortical circuits to converge on similar functional states over a wide range of stimulus inputs.

\section{References}

Anderson JS, Lampl I, Gillespie DC, Ferster D (2000) The contribution of noise to contrast invariance of orientation tuning in cat visual cortex. Science 290:1968-1972.

Arieli A, Sterkin A, Grinvald A, Aertsen A (1996) Dynamics of ongoing activity: explanation of the large variability in evoked cortical responses. Science 273:1868-1871.

Averbeck BB, Latham PE, Pouget A (2006) Neural correlations, population coding and computation. Nat Rev Neurosci 7:358-366.

Bartos M, Vida I, Jonas P (2007) Synaptic mechanisms of synchronized gamma oscillations in inhibitory interneuron networks. Nat Rev Neurosci 8:45-56.

Berens P, Keliris GA, Ecker AS, Logothetis NK, Tolias AS (2008) Comparing the feature selectivity of the gamma-band of the local field potential and the underlying spiking activity in primate visual cortex. Front Syst Neurosci 2:2.

Berman NJ, Maler L (1998) Inhibition evoked from primary afferents in the electrosensory lateral line lobe of the weakly electric fish (Apteronotus leptorhynchus). J Neurophysiol 80:3173-3196.

Börgers C, Kopell N (2003) Synchronization in networks of excitatory and inhibitory neurons with sparse, random connectivity. Neural Comput 15:509-538.

Brunel N (2000) Dynamics of sparsely connected networks of excitatory and inhibitory spiking neurons. J Comput Neurosci 8:183-208.

Brunel N, Hansel D (2006) How noise affects the synchronization properties of recurrent networks of inhibitory neurons. Neural Comput 18:1066-1110.

Bruno RM, Sakmann B (2006) Cortex is driven by weak but synchronously active thalamocortical synapses. Science 312:1622-1627.

Bruno RM, Simons DJ (2002) Feedforward mechanisms of excitatory and inhibitory cortical receptive fields. J Neurosci 22:10966-10975.

Carandini M (2004) Amplification of trial-to-trial response variability by neurons in visual cortex. PLoS Biol 2:E264.

Cardin JA, Carlén M, Meletis K, Knoblich U, Zhang F, Deisseroth K, Tsai LH, Moore CI (2009) Driving fast-spiking cells induces gamma rhythm and controls sensory responses. Nature 459:663-667.

Chalk M, Herrero JL, Gieselmann MA, Delicato LS, Gotthardt S, Thiele A (2010) Attention reduces stimulus-driven gamma frequency oscillations and spike field coherence in V1. Neuron 66:114-125.

Churchland AK, Kiani R, Chaudhuri R, Wang XJ, Pouget A, Shadlen MN (2011) Variance as a signature of neural computations during decision making. Neuron 69:818-831.

Churchland MM, Yu BM, Ryu SI, Santhanam G, Shenoy KV (2006) Neural variability in premotor cortex provides a signature of motor preparation. J Neurosci 26:3697-3712.

Cohen MR, Maunsell JH (2009) Attention improves performance primarily by reducing interneuronal correlations. Nat Neurosci 12:1594-1600.

Constantinople CM, Bruno RM (2011) Effects and mechanisms of wakefulness on local cortical networks. Neuron 69:1061-1068.

de la Rocha J, Doiron B, Shea-Brown E, Josić K, Reyes A (2007) Correlation between neural spike trains increases with firing rate. Nature 448:802-806.
Doiron B, Rinzel J, Reyes A (2006) Stochastic synchronization in finite size spiking networks. Phys Rev E Stat Nonlin Soft Matter Phys 74:030903.

Douglas R, Markram H, Martin K (2004) Neocortex, Ed 5. New York: Oxford UP.

Ecker AS, Berens P, Keliris GA, Bethge M, Logothetis NK, Tolias AS (2010) Decorrelated neuronal firing in cortical microcircuits. Science 327:584-587.

Ermentrout B (1998) Neural networks as spatio-temporal pattern-forming systems. Rep Prog Phys 61:353-430.

Ferezou I, Haiss F, Gentet LJ, Aronoff R, Weber B, Petersen CC (2007) Spatiotemporal dynamics of cortical sensorimotor integration in behaving mice. Neuron 56:907-923.

Ferster D, Miller KD (2000) Neural mechanisms of orientation selectivity in the visual cortex. Annu Rev Neurosci 23:441-471.

Fries P, Reynolds JH, Rorie AE, Desimone R (2001) Modulation of oscillatory neuronal synchronization by selective visual attention. Science 291:1560-1563.

Galán RF, Fourcaud-Trocmé N, Ermentrout GB, Urban NN (2006) Correlation-induced synchronization of oscillations in olfactory bulb neurons. J Neurosci 26:3646-3655.

Ganguly K, Kleinfeld D (2004) Goal-directed whisking increases phaselocking between vibrissa movement and electrical activity in primary sensory cortex in rat. Proc Natl Acad Sci U S A 101:12348-12353.

Gentet LJ, Avermann M, Matyas F, Staiger JF, Petersen CC (2010) Membrane potential dynamics of GABAergic neurons in the barrel cortex of behaving mice. Neuron 65:422-435.

Goldberg JA, Rokni U, Boraud T, Vaadia E, Bergman H (2004) Spike synchronization in the cortex/basal-ganglia networks of parkinsonian primates reflects global dynamics of the local field potentials. J Neurosci 24:6003-6010.

Greenberg DS, Houweling AR, Kerr JN (2008) Population imaging of ongoing neuronal activity in the visual cortex of awake rats. Nat Neurosci 11:749-751.

Gutnisky DA, Dragoi V (2008) Adaptive coding of visual information in neural populations. Nature 452:220-224.

Heiss JE, Katz Y, Ganmor E, Lampl I (2008) Shift in the balance between excitation and inhibition during sensory adaptation of S1 neurons. J Neurosci 28:13320-13330.

Higley MJ, Contreras D (2006) Balanced excitation and inhibition determine spike timing during frequency adaptation. J Neurosci 26:448-457.

Huang X, Troy WC, Yang Q, Ma H, Laing CR, Schiff SJ, Wu JY (2004) Spiral waves in disinhibited mammalian neocortex. J Neurosci 24:9897-9902.

Kanichay RT, Silver RA (2008) Synaptic and cellular properties of the feedforward inhibitory circuit within the input layer of the cerebellar cortex. J Neurosci 28:8955-8967.

Kelly RC, Smith MA, Kass RE, Lee TS (2010) Local field potentials indicate network state and account for neuronal response variability. J Comput Neurosci 29:567-579.

Kerr JN, de Kock CP, Greenberg DS, Bruno RM, Sakmann B, Helmchen F (2007) Spatial organization of neuronal population responses in layer 2/3 of rat barrel cortex. J Neurosci 27:13316-13328.

Kohn A, Smith MA (2005) Stimulus dependence of neuronal correlation in primary visual cortex of the macaque. J Neurosci 25:3661-3673.

Kreiman G, Hung CP, Kraskov A, Quiroga RQ, Poggio T, DiCarlo JJ (2006) Object selectivity of local field potentials and spikes in the macaque inferior temporal cortex. Neuron 49:433-445.

Land PW, Simons DJ (1985) Cytochrome oxidase staining in the rat SmI barrel cortex. J Comp Neurol 238:225-235.

Lewis JE, Maler L (2002) Dynamics of electrosensory feedback: short-term plasticity and inhibition in a parallel fiber pathway. J Neurophysiol 88:1695-1706.

Mazzoni A, Panzeri S, Logothetis NK, Brunel N (2008) Encoding of naturalistic stimuli by local field potential spectra in networks of excitatory and inhibitory neurons. PLoS Comput Biol 4:e1000239.

Miller KD, Pinto DJ, Simons DJ (2001) Processing in layer 4 of the neocortical circuit: new insights from visual and somatosensory cortex. Curr Opin Neurobiol 11:488-497.

Mitchell JF, Sundberg KA, Reynolds JH (2007) Differential attentiondependent response modulation across cell classes in macaque visual area V4. Neuron 55:131-141.

Mitchell JF, Sundberg KA, Reynolds JH (2009) Spatial attention decorre- 
lates intrinsic activity fluctuations in macaque area V4. Neuron 63:879-888.

O'Connor DH, Peron SP, Huber D, Svoboda K (2010) Neural activity in barrel cortex underlying vibrissa-based object localization in mice. Neuron 67:1048-1061.

Oswald AM, Doiron B, Rinzel J, Reyes AD (2009) Spatial profile and differential recruitment of $\mathrm{GABA}_{\mathrm{B}}$ modulate oscillatory activity in auditory cortex. J Neurosci 29:10321-10334.

Papoulis A, Pillai SU (2002) Probability, random variables and stochastic processes, Ed 4. New York: McGraw-Hill.

Petersen CC, Hahn TT, Mehta M, Grinvald A, Sakmann B (2003) Interaction of sensory responses with spontaneous depolarization in layer $2 / 3$ barrel cortex. Proc Natl Acad Sci U S A 100:13638-13643.

Petreanu L, Mao T, Sternson SM, Svoboda K (2009) The subcellular organization of neocortical excitatory connections. Nature 457:1142-1145.

Pinto DJ, Brumberg JC, Simons DJ, Ermentrout GB (1996) A quantitative population model of whisker barrels: re-examining the Wilson-Cowan equations. J Comput Neurosci 3:247-264.

Pinto DJ, Hartings JA, Brumberg JC, Simons DJ (2003) Cortical damping: analysis of thalamocortical response transformations in rodent barrel cortex. Cereb Cortex 13:33-44.

Pouille F, Scanziani M (2001) Enforcement of temporal fidelity in pyramidal cells by somatic feed-forward inhibition. Science 293:1159-1163.

Pouille F, Marin-Burgin A, Adesnik H, Atallah BV, Scanziani M (2009) Input normalization by global feedforward inhibition expands cortical dynamic range. Nat Neurosci 12:1577-1585.

Poulet JF, Petersen CC (2008) Internal brain state regulates membrane potential synchrony in barrel cortex of behaving mice. Nature 454:881-885.

Renart A, de la Rocha J, Bartho P, Hollender L, Parga N, Reyes A, Harris KD (2010) The asynchronous state in cortical circuits. Science 327:587-590.
Reyes AD (2003) Synchrony-dependent propagation of firing rate in iteratively constructed networks in vitro. Nat Neurosci 6:593-599.

Rothschild G, Nelken I, Mizrahi A (2010) Functional organization and population dynamics in the mouse primary auditory cortex. Nat Neurosci 13:353-360.

Simons DJ (1983) Multi-whisker stimulation and its effects on vibrissa units in rat SmI barrel cortex. Brain Res 276:178-182.

Simons DJ, Carvell GE, Hershey AE, Bryant DP (1992) Responses of barrel cortex neurons in awake rats and effects of urethane anesthesia. Exp Brain Res 91:259-272.

Smith MA, Kohn A (2008) Spatial and temporal scales of neuronal correlation in primary visual cortex. J Neurosci 28:12591-12603.

Swadlow HA (2003) Fast-spike interneurons and feedforward inhibition in awake sensory neocortex. Cereb Cortex 13:25-32.

Tiesinga PH, Fellous JM, Salinas E, José JV, Sejnowski TJ (2004) Inhibitory synchrony as a mechanism for attentional gain modulation. J Physiol Paris 98:296-314.

Wehr M, Zador AM (2003) Balanced inhibition underlies tuning and sharpens spike timing in auditory cortex. Nature 426:442-446.

Yizhar O, Fenno LE, Prigge M, Schneider F, Davidson TJ, O'Shea DJ, Sohal VS, Goshen I, Finkelstein J, Paz JT, Stehfest K, Fudim R, Ramakrishnan C, Huguenard JR, Hegemann P, Deisseroth K (2011) Neocortical excitation/inhibition balance in information processing and social dysfunction. Nature 477:171-178.

Yoshimura Y, Callaway EM (2005) Fine-scale specificity of cortical networks depends on inhibitory cell type and connectivity. Nat Neurosci 8:1552-1559.

Zohary E, Shadlen MN, Newsome WT (1994) Correlated neuronal discharge rate and its implications for psychophysical performance. Nature 370:140-143. 